\title{
5G cognitive radio system design with new algorithm asynchronous spectrum sensing
}

\author{
Mohammed Mehdi Saleh', Ahmed A. Abbas², Ahmed Hammoodi ${ }^{3}$ \\ ${ }_{1,2}$ Department of Electrical Engineering, University of Anbar, Iraq \\ ${ }^{3}$ Department of Electrical and Electronic Engineering, University Tun Hussein Onn, Malaysia
}

\section{Article Info \\ Article history:}

Received Feb 2, 2021

Revised Apr 26, 2021

Accepted May 25, 2021

\section{Keywords:}

Cognitive radio

Energy detection

Fifth generation

Ofdm

Sensing time

\begin{abstract}
Due to the rapid increase in wireless applications and the number of users, spectrum scarcity, energy consumption and latency issues will emerge, notably in the fifth generation (5G) system. Cognitive radio (CR) has emerged as the primary technology to address these challenges, allowing opportunist spectrum access as well as the ability to analyze, observe, and learn how to respond to environmental 5G conditions. The CR has the ability to sense the spectrum and detect empty bands in order to use underutilized frequency bands without causing unwanted interference with legacy networks. In this paper, we presented a spectrum sensing algorithm based on energy detection that allows secondary user SU to transmit asynchronously with primary user PU without causing harmful interference. This algorithm reduced the sensing time required to scan the whole frequency band by dividing it into $\mathrm{n}$ sub-bands that are all scanned at the same time. Also, this algorithm allows cognitive radio networks (CRN) nodes to select their operating band without requiring cooperation with licensed users. According to the BER, secondary users have better performance compared with primary users.
\end{abstract}

This is an open access article under the CC BY-SA license.

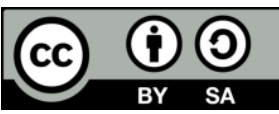

Corresponding Author:

Mohammed Mehdi Saleh

Education College-Qaim

University of Anbar, Ramadi, Iraq

Email: mohammedmhedi@uoanbar.edu.iq

\section{INTRODUCTION}

The exponential rise of mobile smartphones that are rich in media has caused users to opt for ubiquitous mobile broadband networks similar to fixed broadband Internet providers [1]. Many studies have discovered that not all licensed bands are used the majority of the time, implying that the bands are only partially used, as only a few bands are used competitively by many users [2], [3]. With developments in wireless communication, there are pressing issues such as high data rates with higher end-to-end performance requirements and user experience. New challenges arise with the introduction of new applications, such as very low power, low latency and low cost, with a huge number of devices connecting to the network. The dramatic growth of mobile data traffic has prompted the mobile network to move to fifth generation (5G) [4], [5]. The development of 5G mobile networks is becoming one of the main drivers of the information and communications technology industry [6]. 5G networks will provide high data rates and permit communication within a wide bandwidth between the largest number of devices for the longest possible period [7]. In order to provide high-quality services, it is necessary for $5 \mathrm{G}$ wireless networks to incorporate more candidate technologies and to integrate multiple types of functional networks [8]. These technologies include, for example, device-to-device, cognitive radio (CR), software defined networking, massive multi- 
input multi-output and so on [9], [10]. With all these new technologies, the obstacles to the 5G network remain multifaceted, such as the rapid growth of mobile data, traffic and the scarcity of spectrum.

$\mathrm{CR}$ has quickly attracted the interest of industry and academia as one of the promising 5G technologies that can address or partially solve the above challenges. Cognitive radio networks (CRN) are being introduced as a future technology to solve spectrum scarcity with a fast growth of wireless applications, notably in 5G and beyond [11]. In CRN, primary users share their authorized spectrum with secondary users in CRNs provided that the interference caused to the PUs does not exceed an unacceptable level. In order to detect the active PUs, different spectrum sensing methods have been developed to provide more spectrum utilization for SUs without any interference with PUs [12], [13]. In 5G, there are wide frequency bands available for use because there is a wide range of these bands that are not used continuously [14], [15]. The throughput of CRNs can be dramatically improved by providing access to bands unused or exploited by authorized users [16], [17]. CR is known as an intelligent technology capable of tracking, interpreting and making decisions for complex resource distribution and spectrum management [18].

There are parameters to be considered in order to optimize CRN operation in different networking environments. The time available to sense transmission channel and the power of the PUs signal are among these essential parameters affecting the efficiency of spectrum sensing. In addition to this, the parameters affecting signaling and sensing quality are sensing time, energy consumption and throughput of a CRNsSensing time has an effect on energy usage, where increasing the time spent on sensing a single channel will increase energy consumption and reduce the transmitting time of the SUs. Taking into account, noise and channel disruptions such as fading and shadowing lead to decision errors which are measured in terms of false alarms and misdetection probabilities. In this paper, a new spectrum sensing algorithm is proposed based on the energy detection method to improve the throughput of spectrum sensing at different SNR levels. This work focuses on throughput optimization by reducing the required sensing time and increasing the accuracy of SUs allocation without interference with PUs. The contributions of this paper is to provide a general framework for $5 \mathrm{G}$ CR that encompasses:

- Can choose and operate on any frequency band, since this proposed algorithm is not limited to a particular range.

- The proposed CR algorithm does not need any information about PUs, such as the nature of employment and time of operation.

- The proposed algorithm enabled us to reduce the sensing time required to scan the entire working frequency band and increase the CRNs throughput.

- $\quad$ Less time to assign a secondary user with more accuracy in locating the primary user.

- The proposed algorithm has successfully utilized the idle licensed spectrum of PUs without interference between users.

- $\quad$ Based on the above, this algorithm has made it possible to use large frequency bands that could reach $\mathrm{GHz}$.

The remaining sections of the paper are arranged as shown in: section 2 reviews the related work. In section. In section 3, the proposed system model (OFDM system and spectrum sensing) is discussed. Section 4 displays the results of the simulation and discussion. Finally, in section 5 , the conclusion of the paper is presented.

\section{LITERATURE REVIEW}

The new frame structure has been proposed in [19] to predict spectrum scarcity as CR users can estimate channel condition before sensing for accurate sensing outcomes. The simulation results indicate that the performance of SUs is greatly improved by predicting spectrum scarcity. The effects of traffic density, prediction errors and the number of users on performance are also discussed in this paper.

Alom et al. [20] an adaptive threshold model has been proposed to provide a more effective energy detection approach to increase detection efficiency when the SNR is low. The simulation result shows that the detection efficiency using the adaptive threshold was better than the use of the static threshold at the low SNR level.

A new method is presented in [21] to enhance the selection of the sensing channel, which depends on the probabilities of the channels being expected to be inactive. They also present a method for computing prediction probabilities and a new framework structure tested by comparing the obtained results with the standard method. The simulation results of the presented methods show a substantial increase in the throughput.

Bharti et al. [22], optimal power allocation schemes for orthogonal precoded spectral configuration for multi-user system was proposed. The goal of the proposed method is to optimize the total downlink data rate for all users in an OFDM-based CR system, while keeping interference with PUs below predefined 
thresholds. The simulation showed that the proposed method has a much higher data rate for CR system compared to a standard uncoded OFDM and the precoded OFDM power allocation method.

The impact of noise variability on the energy detection performance of signals transmitted by OFDM is reviewed in [23]. In addition, extensive analysis was carried out using simulations of the energy detection method for three different OFDM systems. The simulation results indicate that the trade-off between the evaluated parameters will lead to enhanced the energy detection process of the various OFDM system designs.

Chin [24] performed an estimate of the energy detection parameters in OFDM CR. The paper focused on the detection of energy over undefined noise variances in multipath fading channels. The paper analyzes two detection methods, along with a constant false alarm (CFAR) constant false alarm rate detection probability (CFARDP). The CFARDP is required under different SNRs and functional impairments for a stable sensing outcome in CRs. A not-data-aided (NDA) noise and signal measurement is also provided in the paper. For CRs over multipath fading networks, the proposed NDA estimator utilizes the time-domain CP of OFDM signals. Maximum likelihood (ML) technique and means values and cramer-rao lower bounds (CRLBs) values for noise and signal power estimation were used. Compared to standard energy detection, the approach using these approximate parameters produced a 40-50 percent improvement in the number of sample detections.

Multiplexing orthogonal frequency division with index modulation cognitive radio (OFDM-IM-CR) method was proposed in [25]. The OFDM-IM-CR system was used to check the potential for the use of inactive subcarriers in CR networks for the SU. Three detection methods were proposed for cooperative detection, non-cooperative detection and low-difficulty detection with near-maximum likelihood to enhance detection of PU. In addition, revision of the BER performance assessment for the PU has been recommended.

\section{SYSTEM MODEL}

\subsection{OFDM system model}

A simple block diagram of the CP-OFDM transmitter and the CR spectrum sensing system can be seen in Figure 1. In a discrete time, the equivalent OFDM baseband signals [26], [27] can be represented as shown in:

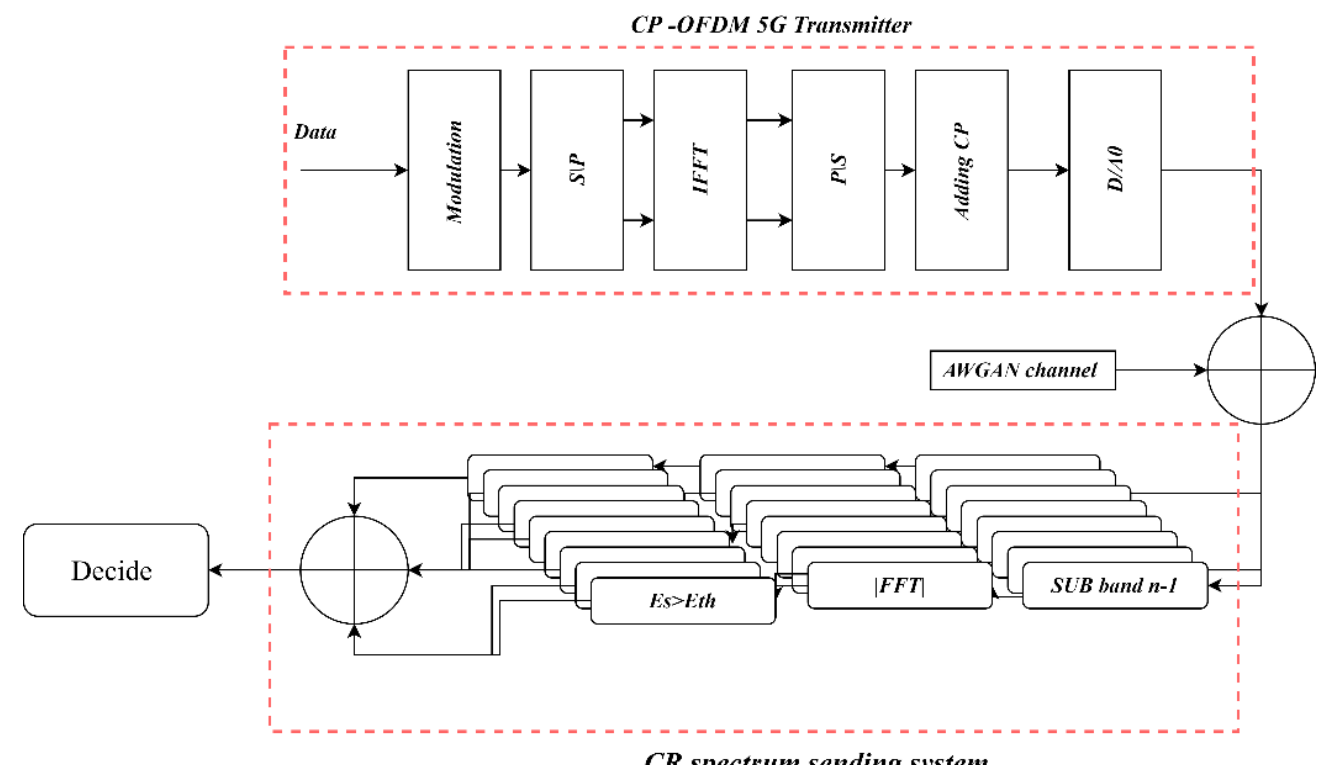

Figure 1. Block diagram of an OFDM transmitter and CR spectrum sensing system

$$
x_{l m}(n)=\sum_{i=0}^{N-1} \mathrm{X}_{l m}(i) e^{j 2 \pi \frac{i n}{N}}
$$

As shown in (1) represents the equivalent OFDM discrete-time signal. Where the transmitter converts the message bits to a series of QAM or PSK symbols that will be mapped into $\mathrm{N}$ parallel streams. 
Let $\mathrm{X}_{l m}(i)$ represent the $1 m$ th transmit symbol of the ith sub-carrier, $\operatorname{lm}=0,1, \ldots, \infty, i=0,1, \ldots, N-1$ and $n=0,1, \ldots$, $N-1$. Note that (1) turns out to be the $N$-point IDFT of $\sum_{i=0}^{N-1} \mathrm{X}_{l m}(i)$ data symbols and can be easily calculated by using an inverse fast fourier transform algorithm. When the channel and the noise effects are not taken into consideration, the OFDM received baseband signal can be represented in the discrete time as seen in (2).

$$
Y_{l m}(i)=\sum_{n=0}^{N-1} y_{l m}(n) e^{-j 2 \pi \frac{i n}{N}}=X_{l m}(i)
$$

Where the received OFDM baseband symbol $y_{l m}(n)=\sum_{i=0}^{N-1} X_{l m}(i) e^{j 2 \pi \frac{i n}{N}}$ from which the transmitted symbol $\mathrm{X}_{l m}(i)$. If the transmitted OFDM signals passing through the AWGN channel with zero mean white noise $\mathrm{e}(\mathrm{n})$, the baseband signal shall be obtained as shown in (3):

$$
r(n)=y(n)+e(n)
$$

CR users find and use the available spectrum opportunities to efficiently utilize the idle licensed spectrum. Depending on the spectrum availability opportunities for CR users, spectrum availability can be represented by a binary assumption model [28] as seen in (4).

$$
r(n)=\left\{\begin{array}{c}
e(t), H_{o} \\
y(t)+e(t), H_{1}
\end{array}\right.
$$

Where $H_{0}$ is a null assumption, that means the PU is inactive on the other side, $H_{1}$ means the PU is active. Neyman-Pearson (NP) detection can also be used to ensure that the probability of a false alarm $\left(P_{f a}\right)$ will not surpass specified levels. Thus, the objective function of the NP decision rule can be written in a simpler way.

$$
Q=P_{d}+g\left(P_{f a}-a\right)
$$

To increase the probability of the detection $P_{d}$, while limiting the $P_{f a}$ at a certain level The variable $g$ in the (5) is the Lagrange multiplier. The NP detector with $P_{f a}$ and $P_{d}$ can be represented as a likelihood ratio test as shown in (6):

$$
\left\{\begin{array}{l}
P_{r}\left(r \mid \mathrm{H}_{1}\right)>\lambda H_{1} \\
P_{r}\left(r \mid H_{0}\right)<\lambda H_{0}
\end{array}\right.
$$

Where $r$ represents the signal received and $\lambda$ the threshold [29].

\subsection{Spectrum sensing model}

In this model, as seen in Figure 2, the peak value $E_{s}$ of the received signal $\mathrm{r}(\mathrm{t})$ in the CR communication range will be compared with the initial threshold $\lambda(7)$. The threshold is calculated when only the $e(t)$ noise signal is present in the communication channel.

$$
\lambda=\max (|\mathrm{FFT}(e(t))|)
$$

If the energy level of the received signal is below the threshold level, the spectrum shall be considered to be available for use by the SUs otherwise it will be considered to be occupied. This model does not require prior knowledge about the primary users and is less complex since it is based on the energy detection method. The frequency band of the received signal B will be divided into sub-bands that will feed the spectrum sensing block. The spectrum sensing block is composed of $\mathrm{n}$ sub-blocks, each of which is in charge of scanning a specific sub-band of the receiving signal. The sub-blocks will make the decision at the same time. By using this algorithm, we will get decisions from $\mathrm{n}$ sub-blocks at the same time, this means that the time required to scan the whole band is $\tau / n$ where $\tau$ represents the sensing time required to scan the entire band without using the proposed algorithm. This algorithm will reduce the sensing time needed to check the whole frequency band by $1 / \mathrm{n}$ vector and increase the detection accuracy, as all these sub-blocks will make decision at one time.

The procedure of the CR algorithm scheme, can be arranged as shown in:

- Begin

- $\quad$ Divide the frequency band B of the received signal $r(t)$ into $\mathrm{n}$ sub-bands (B1, B2,..,Bn).

- Calculatting the initial threshold level $\lambda$ when only a noise signal is present on the channel according to (7). 
- $\quad$ Finding the peak value of the received signal $E_{s}$ within the sub-band by taking the FFT for this received signal and comparing it to the initial threshold level $\lambda$.

- If all signal energy Es levels fall below the initial threshold $\lambda$, the frequency band is marked as available $\mathrm{H}_{0}$.

- $\quad$ Set this band for use by secondary users

- $\quad$ Else, the frequency band is not available $H_{l}$, another band will be chosen for the quest.

- $\quad$ Processing group 2.

- $\quad$ End.

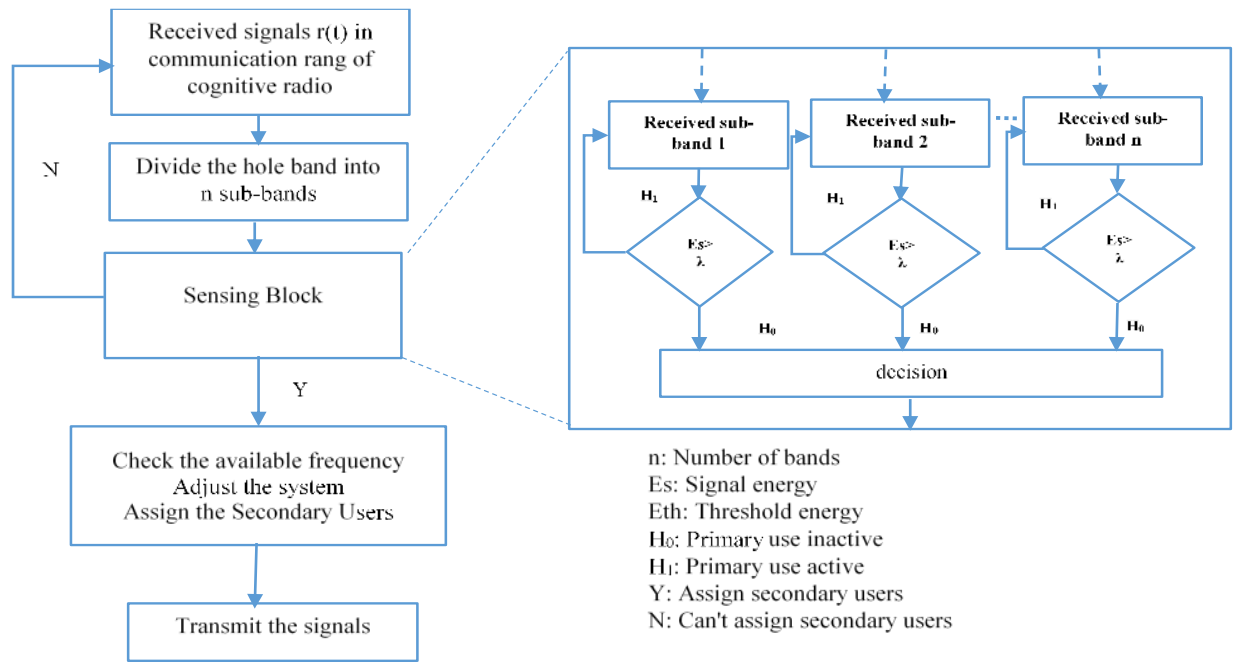

Figure 2. Flow chart of the proposed algorithm

\section{SIMULATION RESULTS AND DISCUSSION}

Assume the working frequency band of the CR system is from $1 \mathrm{~Hz}$ to $10 \mathrm{MHz}$. The frequency band has been divided into 5 sub-bands feeding 5 sub-blocks, where each sub-band is $2 \mathrm{MHz}$ and the number of primary users (PUs) is 20. Next, let's assume the number of idle PUs is 10 and the number of secondary users (SUs) is 10 SUs that will be assigned in the place of the absent PUs. The flow chart in Figure 2 illustrates the ongoing process of the proposed algorithm. It uses an energy detection technique for sensing the PUs to find unused frequencies, and then assigns them to SUs.

The main factor used to assess and determine the overall detection rate of spectrum sensing techniques is the receiver operating characteristics (ROC) curve. ROC has been used to ascertain the proposed theoretical formulas of $P_{d}, P_{f a}$ and $\lambda$ as the first validation scenario. Figure 3 shows the accuracy of the relation between $P_{d}$ and $P_{f a}$ that was tested by evaluating the theoretical ROC energy detection curve for different SNR values. Based on [29] and using the same simulation parameters, the simulation results of the proposed algorithm for $P_{d}, P_{f a}$ and $\lambda$ shows high and accurate compatibility with false alarm conditions and other system parameters. The detection is achieved by comparing the received signal $\mathrm{r}(\mathrm{t})$ to the threshold $\lambda$. Since the transmitted signal, $x(t)$, is presented within the $r(t)$, a successful detection happens when the received signal crossing the threshold, for example $\mathrm{r}(\mathrm{t})>\lambda$. The ability of the detector to detect the target user is measured by the $P_{d}$. The $P_{d}$ is estimated as the ratio between the number of times the $\mathrm{r}(\mathrm{t})$ crossing the threshold $\lambda$ and the number of total trials. But at the other hand, a false alarm happens when the detection indicates that there is a user, but there is really no user. The received signal crosses the threshold when there is only noise present and the error probability of the detector to detect a user when there isn't one is given by $P_{f a}$. The simulation result shows that at $P_{f a} 0.1$ the $P_{d}$ is 0.88666 for $-10 \mathrm{~dB} \mathrm{SNR}$, for $-15 \mathrm{~dB}$ SNR the $P_{d}$ is 0.42349 and for $-20 \mathrm{~dB}$ SNR the $P_{d}$ is 0.24957 for the same $P_{f a}$ which meets our requirement compared with the result in [29].

The periodogram will be used to simulate the spectrum density of the signal. We create random PUs signals in the specific frequency band in this simulation is from $1 \mathrm{~Hz}$ to $10 \mathrm{MHz}$. White gaussian noise has been added to the received signals to simulate real channels. The sensing block measures the energy carried by each symbol, then compares it with the threshold, deciding whether or not the PU is active at this moment. 
In the sensing block, each sub-block scans a specific sub-band to decide if the PU is active or inactive at this time, if the PU inactive, permit the SU to use the vacant spectrum. The performances of the proposed algorithm were checked by comparing the results obtained with the results recorded in [20] and enhanced throughput in [30]. Reducing the sensing time will maximize spectrum utilization, dramatically reduce interference between PUs and SUs, and enable the CRN to achieve the highest throughput. The results of the simulation showed that the proposed algorithm significantly improved CR performance by reducing sensing time and efficiently using licensed bands. As seen in Figures 6-9 secondary users have been effectively allocated to idle PUs frequencies without interference between users. The model also achieved high accuracy in the case of low SNR in the noisy channel at a different threshold level. The following figures are the PU and SU signals for the proposed model simulation. Figure 4, represents the peak values of the primary users signals and the noise signal present in the channel. Figure 5 shows that there are 10 inactive PUs at this moment, then unused frequencies will be assigned to the SUs as seen in Figure 6. Figure 7 shows the PUs signals with blue color and SUs signals with red color. Our model was simulated by using MATLAB 2019 b.

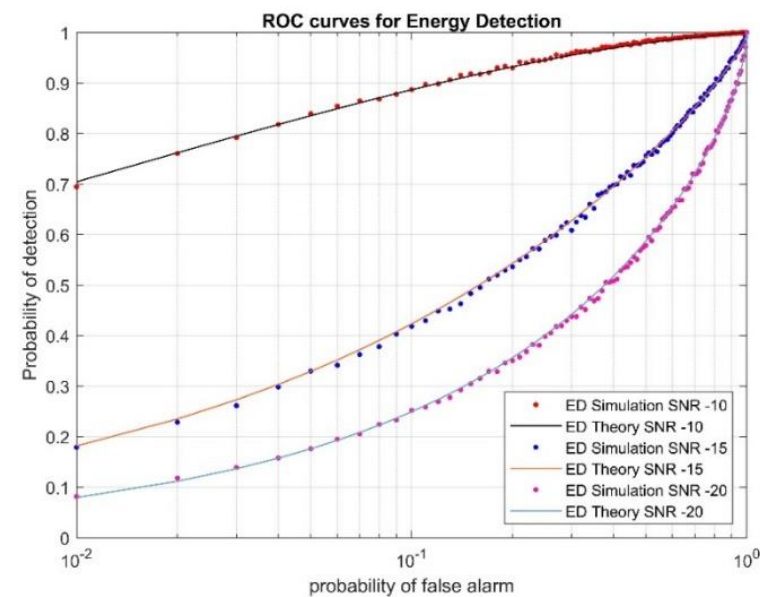

Figure 3. $P_{d}$ compared with $P_{f a}$ using ED ROC curves with various SNR values

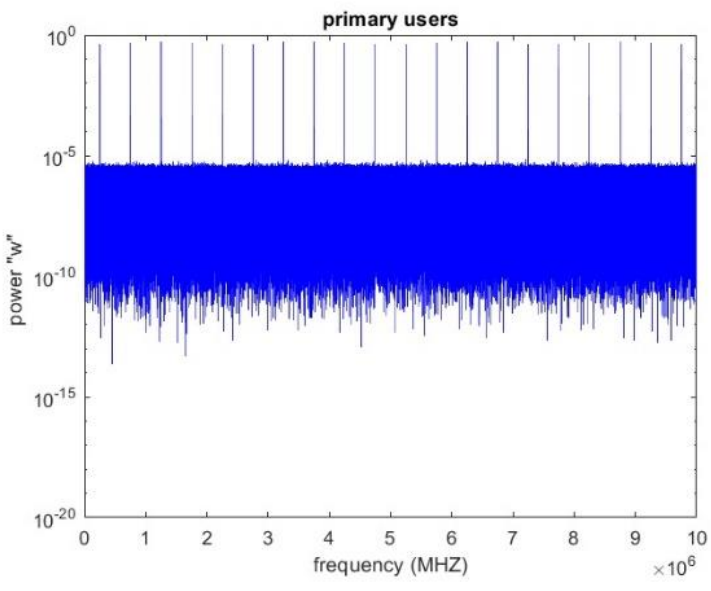

Figure 4. The PUs signals for 20 users

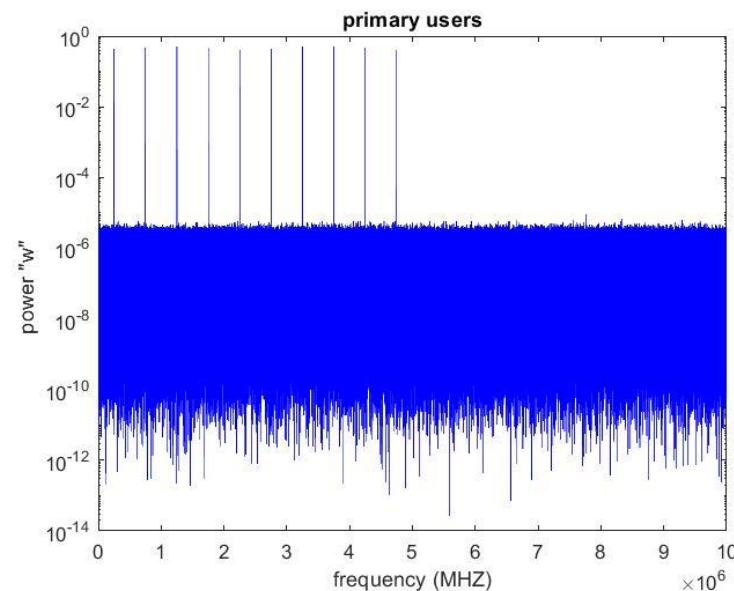

Figure 5. The PUs signals with 10 idle users 


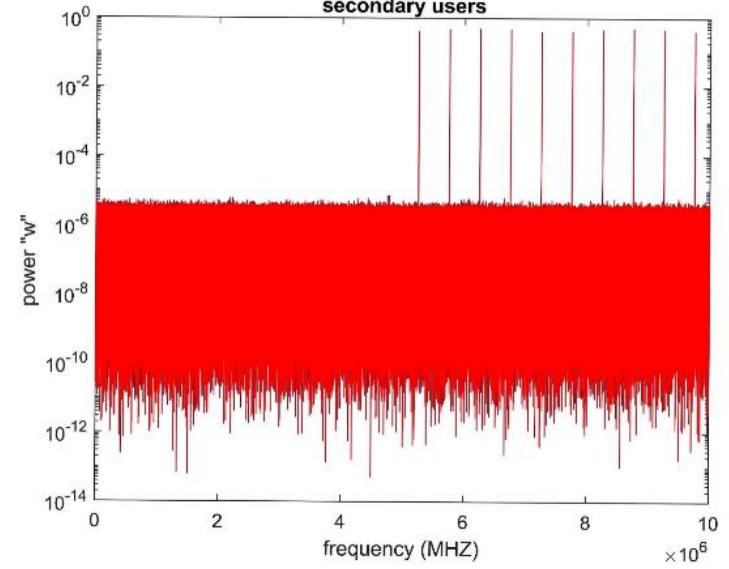

Figure 6. The SUs signals assigned to the empty frequencies

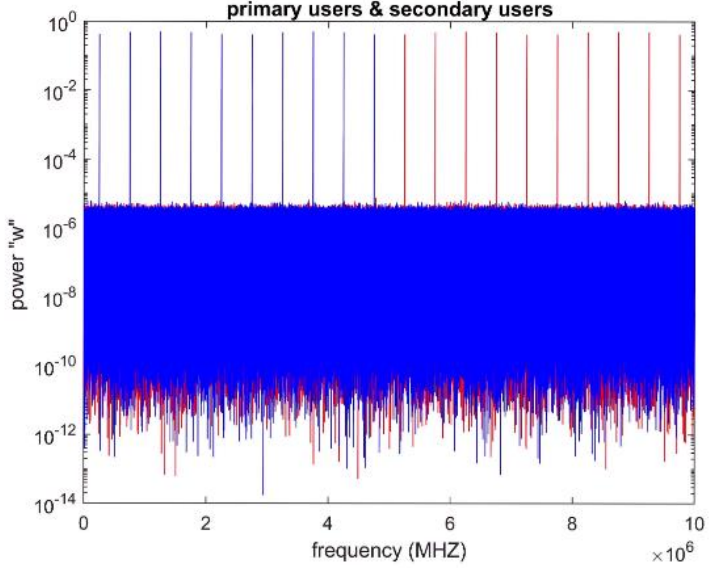

Figure 7. The PUs signals with blue color and SUs signals with red color

Figure 8 illustrates downlink throughput per user and Figure 9 is BER per user when using the proposed CR algorithm. Where UE1 represents the primary user and UE2 represents the secondary user. The secondary user UE2 used the primary user UE1 band in their absence and successfully achieved throughput without interference. According to the BER the secondary user achieved better performance compared with primary users, whereas the throughput for primary users is more achievable to use in the algorithm as shown in figure 8 and 9. We simulate the 5G environment by using MATLAB and LabVIEW, then input the simulation result from LabVIEW to AGW devices and display the output by using MATLAB.

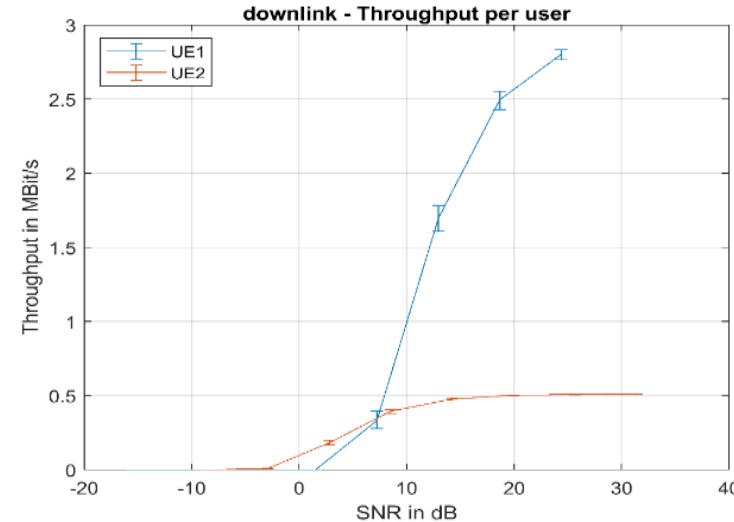

Figure 8. Downlink throughput per user when used the proposed algorithm for $\mathrm{CR}$

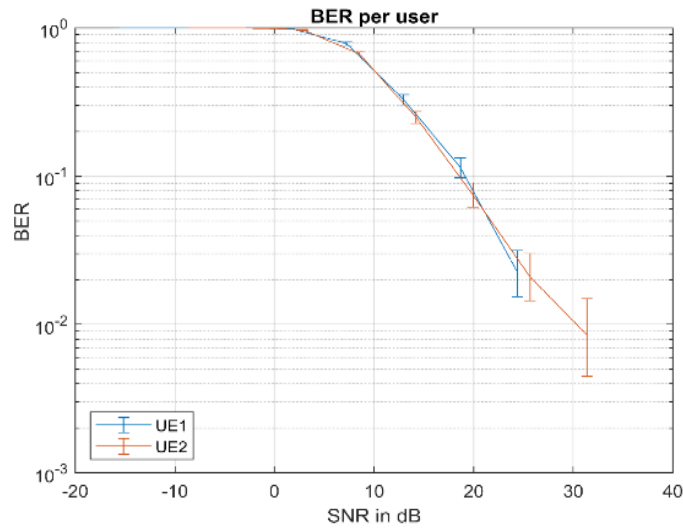

Figure 9. BER per user when used the proposed algorithm for CR

\section{CONCLUSION}

The results of the simulations show that with different levels of SNR of the primary users $-10 \mathrm{~dB},-15 \mathrm{~dB}$, and $20 \mathrm{~dB}$, the optimum time has been reached to achieve the highest throughput while keeping the probability of detection within the required limit. The proposed algorithm successfully reduces the sensing time needed to assign SUs in a $5 \mathrm{G}$ system by $1 / n$ vector. According to the BER, the secondary user achieved better performance compared with primary user, whereas the throughput for the primary user is more achievable to use in the algorithm. Using this algorithm, we are able to detect the empty spectrum, which is initialized and dynamically allocated to the unlicensed user, and once the licensed user looks for its assigned band, the SU transfers its band to any other available band. 


\section{REFERENCES}

[1] I. Ali, M. H. Jamaluddin, M. R. Kamarudin, A. Gaya, and R. Selvaraju, "Wideband and high gain dielectric resonator antenna for 5g applications," Bulletin of Electrical Engineering and Informatics, vol. 8, no. 3, pp. 137141, 2019, doi: 10.11591/eei.v8i3.1592.

[2] J. Divya Lakshmi and L. Rangaiah, "Cognitive radio principles and spectrum sensing," International Journal of Engineering and Advanced Technology (IJEAT), vol. 8, no. 6, 2019, pp. 4294-4298, doi: 10.35940/ijeat.F8884.088619.

[3] M. J. Marcus, "Spectrum Policy and Regulatory Issues," in IEEE Wireless Communications, vol. 24, no. 2, pp. 4-5, April 2017, doi: 10.1109/MWC.2017.7909088.

[4] J. F. Monserrat, G. Mange, V. Braun, H. Tullberg, G. Zimmermann, and Ö. Bulakci, "METIS research advances towards the 5G mobile and wireless system definition," EURASIP Journal on Wireless Communications and Networking, vol. 2015, no. 1, pp. 1-16, 2015, doi: 10.1186/s13638-015-0302-9.

[5] M. Y. Zeain, et al., "Design of a wideband strip helical antenna for 5g applications," Bulletin of Electrical Engineering and Informatics, vol. 9, no. 5, pp. 1958-1963, 2020, doi: 10.11591/eei.v9i5.2055.

[6] M. Agiwal, A. Roy and N. Saxena, "Next Generation 5G Wireless Networks: A Comprehensive Survey," in IEEE Communications Surveys and Tutorials, vol. 18, no. 3, pp. 1617-1655, thirdquarter 2016, doi: 10.1109/COMST.2016.2532458.

[7] C. Jiang, H. Zhang, Y. Ren, Z. Han, K. -C. Chen and L. Hanzo, "Machine Learning Paradigms for Next-Generation Wireless Networks," in IEEE Wireless Communications, vol. 24, no. 2, pp. 98-105, April 2017, doi: 10.1109/MWC.2016.1500356WC.

[8] A. H. Fadel, H. H. Razzaq, and S. A. Mostafa, "A low complexity partial transmit sequence approach based on hybrid segmentation scheme," Bulletin of Electrical Engineering and Informatics (BEEI), vol. 9, no. 6, pp. 23712379, 2020, doi: 10.11591/eei.v9i6.2520.

[9] M. Shafi, et al., "5G: A Tutorial Overview of Standards, Trials, Challenges, Deployment, and Practice," in IEEE Journal on Selected Areas in Communications, vol. 35, no. 6, pp. 1201-1221, June 2017, doi: 10.1109/JSAC.2017.2692307.

[10] M. Q. Taha, Z. H. Ali, and A. K. Ahmed, "Two-level scheduling scheme for integrated 4G-WLAN network," International Journal of Electrical and Computer Engineering, vol. 10, no. 3, 2019, p. 2633, doi: 10.11591/ijece.v10i3.pp2633-2643.

[11] S. Sasipriya and R. Vigneshram, "An overview of cognitive radio in 5G wireless communications," 2016 IEEE International Conference on Computational Intelligence and Computing Research (ICCIC), 2016, pp. 1-5, doi: 10.1109/ICCIC.2016.7919725.

[12] P. T. Sivagurunathan, P. Ramakrishnan, and N. Sathishkumar, "Recent paradigms for efficient spectrum sensing in cognitive radio networks: Issues and challenges," in Journal of Physics: Conference Series, 2021, vol. 1717, no. 1, p. 012057, doi: 10.1088/1742-6596/1717/1/012057.

[13] A. J. Onumanyi, A. M. Abu-Mahfouz, and G. P. Hancke, "A comparative analysis of local and global adaptive threshold estimation techniques for energy detection in cognitive radio," Physical Communication, vol. 29, 2018, pp. 1-11, doi: 10.1016/j.phycom.2018.04.008.

[14] M. Jia, X. Gu, Q. Guo, W. Xiang and N. Zhang, "Broadband Hybrid Satellite-Terrestrial Communication Systems Based on Cognitive Radio toward 5G," in IEEE Wireless Communications, vol. 23, no. 6, pp. 96-106, December 2016, doi: 10.1109/MWC.2016.1500108WC.

[15] A. A. Jabber and R. H. Thaher, "Compact tri-band T-shaped frequency reconfigurable antenna for cognitive radio applications," Bulletin of Electrical Engineering and Informatics (BEEI), vol. 9, no. 1, pp. 212-220, 2020, doi: 10.11591/eei.v9i1.1708.

[16] X. Liu, F. Li and Z. Na, "Optimal Resource Allocation in Simultaneous Cooperative Spectrum Sensing and Energy Harvesting for Multichannel Cognitive Radio," in IEEE Access, vol. 5, pp. 3801-3812, 2017, doi: 10.1109/ACCESS.2017.2677976.

[17] D. A. G. Ramirez, C. Hernandez, and F. Martinez, "Throughput in cooperative wireless networks," Bulletin of Electrical Engineering and Informatics, vol. 9, no. 2, pp. 698-706, 2020, doi: 10.11591/eei.v9i2.2025.

[18] R. Li, et al., "Intelligent 5G: When Cellular Networks Meet Artificial Intelligence," in IEEE Wireless Communications, vol. 24, no. 5, pp. 175-183, October 2017, doi: 10.1109/MWC.2017.1600304WC.

[19] J. Yang and H. Zhao, "Enhanced Throughput of Cognitive Radio Networks by Imperfect Spectrum Prediction," in IEEE Communications Letters, vol. 19, no. 10, pp. 1738-1741, Oct. 2015, doi: 10.1109/LCOMM.2015.2442571.

[20] M. Z. Alom, T. K. Godder, M. N. Morshed and A. Maali, "Enhanced spectrum sensing based on Energy detection in cognitive radio network using adaptive threshold," 2017 International Conference on Networking, Systems and Security (NSysS), 2017, pp. 138-143, doi: 10.1109/NSysS.2017.7885815.

[21] P. Thakur, A. Kumar, S. Pandit, G. Singh, and S. N. Satashia, "Performance analysis of cognitive radio networks using channel-prediction-probabilities and improved frame structure," Digital Communications and Networks, vol. 4, no. 4, 2018, pp. 287-295, doi: 10.1016/j.dcan.2017.09.012.

[22] M. R. Bharti and D. Ghosh, "Power allocation for multiuser precoded OFDM cognitive radio," Physical Communication, vol. 30, pp. 287-295, 2018, doi: 10.1016/j.phycom.2018.09.002.

[23] J. Lorincz, I. Ramljak, and D. Begušić, "A review of the noise uncertainty impact on energy detection with different OFDM system designs," Computer Communications, vol. 148. pp. 185-207, 2019, doi: 10.1016/j.comcom.2019.09.013.

[24] W. -L. Chin, "On the Noise Uncertainty for the Energy Detection of OFDM Signals," in IEEE Transactions on Vehicular Technology, vol. 68, no. 8, pp. 7593-7602, Aug. 2019, doi: 10.1109/TVT.2019.2920142. 
[25] J. Li, Y. Peng, Y. Yan, X. Jiang, H. Hai and M. Zukerman, "Cognitive Radio Network Assisted by OFDM With Index Modulation," in IEEE Transactions on Vehicular Technology, vol. 69, no. 1, pp. 1106-1110, Jan. 2020, doi: 10.1109/TVT.2019.2951606.

[26] O. Okoyeigbo, K. Okokpujie, E. Noma-Osaghae, C. U. Ndujiuba, O. Shobayo, and A. Jeremiah, "Comparative study of MIMO-OFDM channel estimation in wireless systems," International Review on Modelling and Simulations, vol. 11, no. 3, p. 158, 2018, doi: 10.15866/iremos.v11i3.13884.

[27] P. Manhasa and M. K. Sonib, "Performance of ofdm system under different fading channels and coding," Bulletin of Electrical Engineering and Informatics, vol. 6, no. 1, pp. 54-61, 2017, doi: 10.11591/eei.v6i1.591.

[28] E. Axell and E. G. Larsson, "Optimal and Sub-Optimal Spectrum Sensing of OFDM Signals in Known and Unknown Noise Variance," in IEEE Journal on Selected Areas in Communications, vol. 29, no. 2, pp. 290-304, February 2011, doi: 10.1109/JSAC.2011.110203.

[29] H. A. O. Selim, A. S. Dessouki, and H. Y. M. Soliman, "Verification analysis for the reliable analytical multi-taper detector in next generation network," Bulletin of Electrical Engineering and Informatics, vol. 9, no. 4, pp. 14861496, 2020, doi: 10.11591/eei.v9i4.2229.

[30] T. C. Thanuja, K. A. Daman and A. S. Patil, "Optimized Spectrum sensing Techniques for Enhanced Throughput in Cognitive Radio Network," 2020 International Conference on Emerging Smart Computing and Informatics (ESCI), 2020, pp. 137-141, doi: 10.1109/ESCI48226.2020.9167576.

\section{BIOGRAPHIES OF AUTHORS}

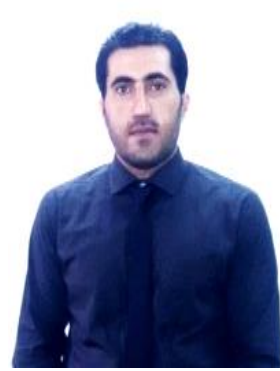

Mohammed Mehdi Saleh received the bachelor's degree in Electrical Engineering from University of Anbar, Iraq in 2009 and the master degree in Electronics and communication Engineering (SSP) from Osmania University, India in 2014. He has served as a teaching assistant for the following undergraduate courses taught at Al-maaref University College: Information and Coding Theory, Computer Network, Real Time Signal Processing, OFC and he is presently teaching staff at University of Anbar. His main interests are in 5G, WSNs.

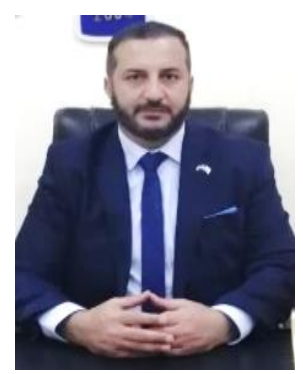

Ahmed A. Abbas is A head of Electrical Engineering Department, College of Engineering, University of Anbar from 2018 till now. He received the B.Sc. degree in Electrical Engineering/ University of Technology in 1994, he received MS.c. in communication Engineering/University of Technology in 2005 and he received $\mathrm{PhD}$ in communication Engineering/Ankara Yildırım Beyazıt University in 2017. Dr. Ahmed is researcher in optical wave propagation through random media.

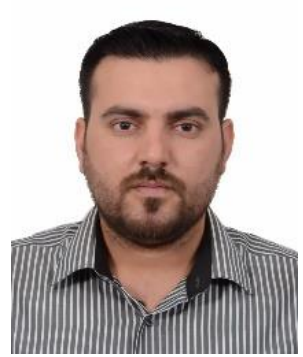

Ahmed Hammoodi received the Engineering degree in computer communication and electrical engineering in Baghdad, Iraq, in 2007, and the M.Sc. degree in wireless communications from University De Mont Fort, Leicester, U.K., in 2015. He is currently pursuing the Ph.D. degree in wireless communications for 5G with the Faculty of Electrical and Electronic Engineering, University Tun Hussein Onn Malaysia, Malaysia. In 2008, he joined Iraqna Company, which specializes in telecommunications. He was with Motorola, Iraq, which specializes in transmission line modeling. His research activities focus on studying how new physical layer technologies, such as advanced multicarrier waveforms, can enhance the coexistence of $5 \mathrm{G}$ communications, such as $\mathrm{D} 2 \mathrm{D}$, M2M, or the IoT, with legacy LTE networks. 